\title{
Optical, Perceptual and Adaptational Implications of different Presbyopic corrections
}

\section{Implicaciones ópticas, perceptivas y de adaptación de diferentescorrecciones Presbicia}

\author{
Aiswaryah Radhakrishnan*, Carlos Dorronsoros, Susana Marcos ${ }^{s}$ \\ Department of Visual Optics and Biophotonics, Instituto de Óptica \\ "Daza de Valdés", Consejo Superior de Investigaciones Científicas, Madrid, Spain \\ ${ }^{*}$ E-mail: raishu85@gmail.com \\ S: miembro de SEDOPTICA / SEDOPTICA member \\ Received / Recibido: 26/06/2019 \\ .Accepted / Aceptado: 15/04/2020
}

DOI: 10.7149/OPA.53.2.51026

\begin{abstract}
:
This publication represents the summary of results of the doctoral thesis Presbyopia Corrections: Optical, Perceptual and Adaptational Implications presented at the Universidad Complutense de Madrid, Spain. Presbyopia, the physiological inability of the aging crystalline lens to accommodate for objects at near distance, can be treated using a multitude of optical corrections. Systematic evaluation of the visual system's ability to interact with these corrections will help in optimization of their performance. In this thesis we studied optical and perceptual performance of different presbyopic corrections such as alternating vision, monovision and simultaneous vision and the effect of adaptation on perceptual performances. We used custom-developed adaptive optics setup to measure and correct ocular aberrations and projected manipulated images simulating pure simultaneous vision corrections of different far/near energy profiles and near additions. We also developed and validated an on-bench and a hand-held simultaneous vision simulator to optically simulate pure or segmented simultaneous vision corrections. Psychophysical methods were employed to study the changes in perceptual quality and after-effects of adaptation. We performed numerical simulations to predict perceptual performance from the ocular aberrations of the subjects. We demonstrated that mechanism of adaptation to simultaneous vision is similar to that of blur adaptation, influenced mostly by retinal image contrast and that systematic changes in visual and perceptual performance influenced by multifocal design and testing distance. The ocular optics of the subject's eye correlated significantly with visual performance and was associated with the intersubject variability in performance. We found that a cyclopean locus for perception and adaptation, in subjects with different blur magnitude between eyes, influenced by the eyes with better optical quality. The internal code for blur was also influenced by the eye with better optical quality, both in orientation and magnitude. Our results confirm that the existing optical solutions should be chosen based on the subjective needs and the ocular optics would be an ideal starting point to customize optical solutions of presbyopia for optimal performance. We also demonstrated the usefulness of rapid assessment of perception to multifocal designs using the handheld, seethrough simultaneous vision simulator.
\end{abstract}

Key words: presbyopia, simultaneous vision, monovision, interocular blur difference, retinal image quality, blur adaptation, cyclopean locus, neural PSF, adaptive optics, vision simulator 


\section{References}

[1] W. N. Charman, "Developments in the correction of presbyopia II: surgical approaches," Ophthalmic Physiol Opt 34, 397-426 (2014).

[2] W. N. Charman, "Developments in the correction of presbyopia I: spectacle and contact lenses," Ophthalmic Physiol Opt 34, 8- 29 (2014).

[3] T. Kohnen, "Multifocal IOL technology: a successful step on the journey toward presbyopia treatment," J Cataract Refract Surg 34, 2005 (2008).

[4] M. A. Garland, "Monovision and related techniques in the management of presbyopia," CLAO J 13, 179-181 (1987).

[5] B. J. Evans, "Monovision: a review," Ophthalmic Physiol Opt 27, 417-439 (2007).

[6] R. Montes-Mico and J. L. Alio, "Distance and near contrast sensitivity function after multifocal intraocular lens implantation," J Cataract Refract Surg 29, 703-711 (2003).

[7] S. Cillino, A. Casuccio, F. Di Pace, R. Morreale, F. Pillitteri, G. Cillino, and G. Lodato, "One-year outcomes with newgeneration multifocal intraocular lenses," Ophthalmology 115, 1508-1516 (2008).

[8] B. Cochener, A. Lafuma, B. Khoshnood, L. Courouve, and G. Berdeaux, "Comparison of outcomes with multifocal intraocular lenses: a meta-analysis," Clin Ophthalmol 5, 45-56 (2011).

[9] M. Leyland and E. Zinicola, "Multifocal versus monofocal intraocular lenses in cataract surgery: a systematic review," Ophthalmology 110, 1789-1798 (2003).

[10] R. Bellucci, "Multifocal intraocular lenses," Curr Opin Ophthalmol 16, 33-37 (2005).

[11] K. Kamiya, K. Hayashi, K. Shimizu, K. Negishi, M. Sato, H. Bissen-Miyajima, C. Survey Working Group of the Japanese Society of, and S. Refractive, "Multifocal intraocular lens explantation: a case series of 50 eyes," Am J Ophthalmol 158, 215-220, E1 (2014).

[12] M. van der Mooren, R. Steinert, F. Tyson, M. J. Langeslag, and P. A. Piers, "Explanted multifocal intraocular lenses," J Cataract Refract Surg 41, 873-877 (2015).

[13] P. de Gracia, C. Dorronsoro, A. Sanchez-Gonzalez, L. Sawides, and S. Marcos, "Experimental simulation of simultaneous vision," Investigative ophthalmology \& visual science 54, 415-422 (2013).

[14] P. Artal, L. Chen, E. J. Fernandez, B. Singer, S. Manzanera, and D. R. Williams, "Neural compensation for the eye's optical aberrations," J Vis 4, 281-287 (2004).

[15] L. Sawides, P. de Gracia, C. Dorronsoro, M. Webster, and S. Marcos, "Adapting to blur produced by ocular high-order aberrations," J Vis 11(2011).

[16] L. Sawides, P. de Gracia, C. Dorronsoro, M. A. Webster, and S. Marcos, "Vision is adapted to the natural level of blur present in the retinal image," PloS one 6, e27031 (2011).

[17] L. Sawides, C. Dorronsoro, A. M. Haun, E. Peli, and S. Marcos, "Using pattern classification to measure adaptation to the orientation of high order aberrations," PloS one 8, e70856 (2013).

[18] P. de Gracia, C. Dorronsoro, and S. Marcos, "Multiple zone multifocal phase designs," Optics letters 38, 3526-3529 (2013).

[19] K. Fisher, "Presbyopic visual performance with modified monovision using multifocal soft contact lenses," International Contact Lens Clinic 24, 10 (1997).

[20] K. R. Johannsdottir and L. B. Stelmach, "Monovision: a review of the scientific literature," Optom Vis Sci 78, 646-651 (2001).

[21] A. Radhakrishnan, C. Dorronsoro, L. Sawides, and S. Marcos, "Short-term neural adaptation to simultaneous bifocal images," PloS one 9, e93089 (2014).

[22] C. Dorronsoro, A. Radhakrishnan, P. de Gracia, L. Sawides, and S. Marcos, "Perceived image quality with simulated segmented bifocal corrections," Biomedical optics express 7, 4388-4399 (2016).

[23] A. Radhakrishnan, C. Dorronsoro, and S. Marcos, "Differences in visual quality with orientation of a rotationally asymmetric bifocal intraocular lens design," J Cataract Refract Surg 42, 1276-1287 (2016).

[24] M. Vinas, C. Dorronsoro, V. Gonzalez, D. Cortes, A. Radhakrishnan, and S. Marcos, "Testing vision with angular and radial multifocal designs using Adaptive Optics," Vision Res (2016).

[25] C. Dorronsoro, A. Radhakrishnan, J. Alonso-Sanz, D. Pascual, M. Velasco-Ocana, P. Perez-Merino, and S. Marcos, "Portable simultaneous vision device to simulate multifocal corrections " Optica In Press (2016).

[26] E. J. Fernandez, L. Vabre, B. Hermann, A. Unterhuber, B. Povazay, and W. Drexler, "Adaptive optics with a magnetic deformable mirror: applications in the human eye," Opt Express 14, 8900-8917 (2006).

[27] C. Dorronsoro and S. Marcos, "Instrument for the simulation of multifocal ophthalmic corrections," (2009).

[28] M. A. Webster, M. A. Georgeson, and S. M. Webster, "Neural adjustments to image blur," Nature neuroscience 5, 839840 (2002).

[29] M. A. Webster, "Adaptation and visual coding," J Vis 11(2011).

[30] Z. Wang, A. C. Bovik, H. R. Sheikh, and E. P. Simoncelli, "Image quality assessment: from error visibility to structural similarity," IEEE transactions on image processing : a publication of the IEEE Signal Processing Society 13, 600-612 (2004).

[31] H. L. Roth, A. N. Lora, and K. M. Heilman, "Effects of monocular viewing and eye dominance on spatial attention," Brain 125, 2023-2035 (2002).

[32] W. H. Ehrenstein and A. Ehrenstein, Psychophysical methods: In modern techniques in neuroscience research (Springer, Berlin, 1999).

[33] D. H. Brainard, "The Psychophysics Toolbox," Spatial vision 10, 433-436 (1997).

[34] D. G. Pelli and B. Farell, Psychophysical methods: In Handbook of Optics (Mc Graw Hill Publishers, New York, 1995).

[35] J. A. Phipps, A. J. Zele, T. Dang, and A. J. Vingrys, "Fast psychophysical procedures for clinical testing," Clin Exp Optom 84, $264-269$ (2001).

[36] A. J. Ahumada, Jr., "Classification image weights and internal noise level estimation," J Vis 2, 121-131 (2002).

[37] M. P. Eckstein and A. J. Ahumada, Jr., "Classification images: a tool to analyze visual strategies," J Vis 2, 1x (2002).

Opt. Pura Apl. 53 (2) 1-15 (2020) 


\section{Introduction}

Near vision restoration in a presbyope is challenging even with the availability of optical solutions such as alternating vision, monovision and simultaneous vision corrections [1-3]. Unlike in alternating vision [2], where distinct portions for far, near (and intermediate) corrections are given in spectacles resulting in clear vision at any one distance, monovision corrects one eye for far vision and the other eye for near vision resulting in binocular vision problems [4,5]. Multifocal optical solutions (contact lenses or intraocular lenses), uses different parts of the pupil of the same eye for corrections at far and near distances producing superimposed blurred and sharp images at retina also known as simultaneous vision [1]. Compared to a monofocal correction at distance, simultaneous vision corrections degrade distance visual performance while augmenting near vision [6-9]. The brain counteracts this complex blur by the suppression of either distance or near image, eventually adapting to the other. However, not all subjects can adapt to this complex blur, as indicated by the explantation rate with multifocal IOLs [10-12]. A better understanding of the optical properties of these corrections and how they influence the visual perception and adaptation will help in designing better optical solutions for presbyopia, which provides an optical visual and perceptual quality at all distances.

Clinical studies comparing visual function on patients implanted with multifocal intraocular lenses or fitted by contact lenses of various designs [11], the impact of simultaneous images on visual performance, and to what extent patients can adapt to simultaneous vision corrections have been hardly explored. de Gracia et al, used a custom-developed simultaneous vision simulator, found that the amount of near addition affected visual acuity differently, with additions around $2 \mathrm{D}$ causing the largest degradation for far vision in pure simultaneous vision [13]. However, multifocal lenses with angular design introduce oriented blur in the retina. Recent reports have provided evidence that observers are selectively adapted to the amount and orientation of blur produced by their ocular aberrations [14-17]. Two independent studies showed that images blurred with the observer's own Point Spread Function (PSF) appeared sharper than images blurred by rotated versions of the same PSF and the images blurred by the observer's PSF was chosen as optimally focused from a series of images. In fact, images blurred by the subject's own aberrations produce no aftereffects and the measured the internal code for blur in subjects was highly correlated with the subject's own PSF [17]. A computational study on diffraction-limited optics showed that the image quality of a multifocal lens of angular design produced superior image quality than a multifocal lens of radial design. In addition, the number of multifocal zones affected the image quality differently [18].

Few studies show that in monovision and modified monovision corrections $[5,19]$ the contrast sensitivity and high contrast visual acuity were little affected in presbyopic subjects. Also, pre-presbyopes adapt more easily to monovision corrections than presbyopes [20]. While the optical design is a key factor influencing visual performance, strong inter-subject differences in preference to monocular presbyopic corrections have been demonstrated [13,21-25] and the additional need to balance binocular behavior makes it particularly challenging to identify or customize an optimal presbyopic correction for a subject. Some of these issues can be addressed by demonstrating vision with multifocal correction to patients, preoperatively.

In this thesis, we designed experiments and utilized/modified optical setups to systematically evaluate subjective preferences, performance and adaptation to pure simultaneous vision, oriented blur and interocular differences in blur. We also developed open-field binocular, portable vision simulator that could be applied in clinical practice.

\section{Setups}

\section{2.a. Adaptive Optics setup}

We used a custom-developed adaptive optics (AO) system to measure and correct ocular aberrations of the subjects' eye. The principal components and different channels of the adaptive optics (AO) system is shown in figure 1. The illumination channel consists of the Super-Luminescent-Diode coupled with an 
optical fiber (Superlum, Ireland) emitting a collimated beam of about $1 \mathrm{~mm}$ at $827 \mathrm{~nm}$. The measurement channel has a Badal system, a Shack-Hartmann wavefront sensor with a CDD camera (HASO 32 OEM, Imagine Eyes, France) and a magnetic deformable mirror with 52 actuators (MIRAO 52, Imagine Eyes, France). The collimated beam of light from the SLD entering the eye is reflected by the retina and passes through the Badal system, the electromagnetic membrane deformable mirror and then is focused on to the Shack-Hartmann wavefront sensor. In an artificial eye, the RMS was reduced by over $97 \%$ and in human eyes the correction efficiency was about $89 \%$. These components could be controlled simultaneously using an interactive software custom-developed in Visual C++ (Microsoft Inc).

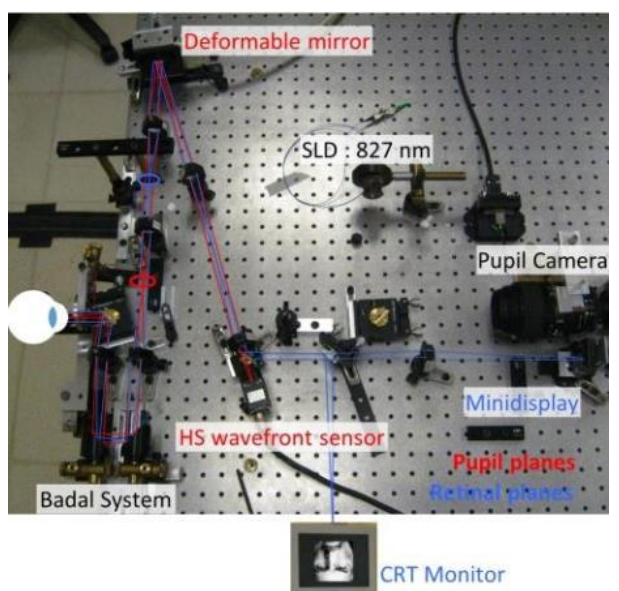

Figure 1: Adaptive Optics setup with principle components highlighted and the retinal (in blue) and pupil planes (in red)

The AO system has two psychophysical channels: A minidisplay (LE400, LiteEye Systems, France) and a CRT monitor (Mitsubhishi Inc..,.) incorporated beyond the wavefront sensor allowing image manipulation for psychophysical tasks under controlled optical aberrations. A flip-mounted mirror is used for selecting either of the channels. The monitor was controlled using a ViSaGe platform (Cambridge Research Systems, UK) for both stimulus presentation and gamma correction. A CCD camera (TELI, Toshiba) with an objective placed conjugate to the artificial pupil allows for continuous pupil monitoring during aberration measurement/correction and psychophysical measurements.

\section{2.b. On-bench Simultaneous Vision simulator}

The first generation simultaneous vision simulator was developed in our lab to assess visual performance with pure simultaneous vision [13]. This was achieved by using a beam splitter to send the stimuli through two independent Badal channels and recombining the images at the pupil plane [26].

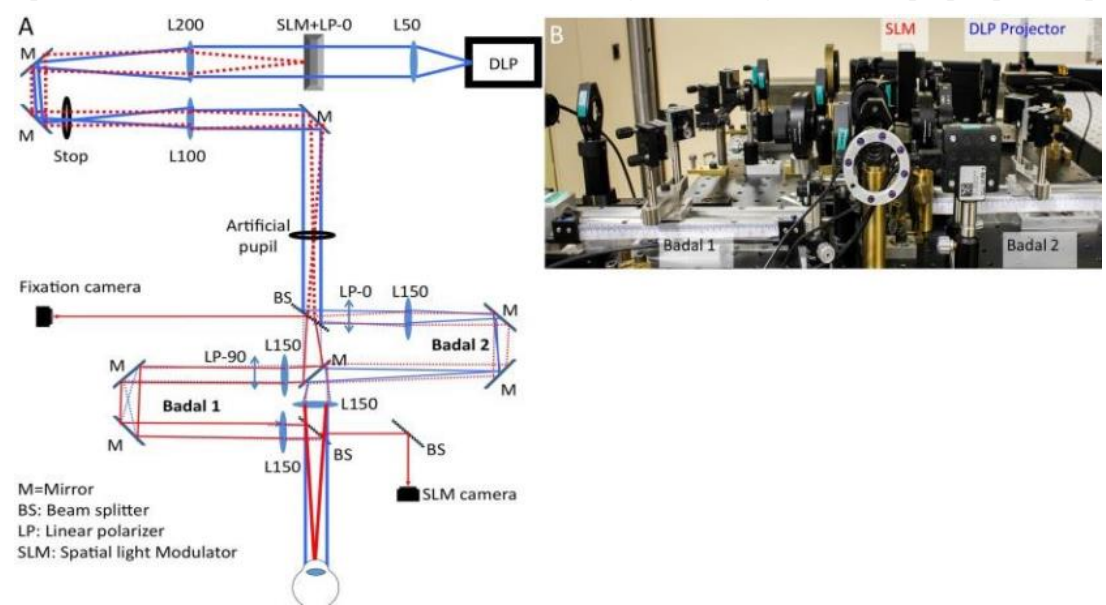

Figure 2: (A) Schematic diagram of the modified SimVis showing the retinal planes (in blue) and pupil planes (in red) for Bad al channels 1 and 2. It also shows the principal components of the system (DLP, SLM and Badal systems) (B) Photograph of the modified SimVis setup highlighting the principle components

To optically simulate refractive multifocal lenses (angular or radial designs with different far-near energy ratios) we modified the simultaneous vision simulator by incorporating a spatial light modulator (LC2002, Holoeye Inc...) and improved the spatial extent of test stimuli that could be presented through the system 
for psychophysical tasks. A schematic representation of the modified simultaneous vision simulator (SimVis) is shown in figure 2A highlighting the pupil and retinal planes of interest corresponding to each Badal channel. An artificial pupil was introduced with a pinhole (x1 magnification factor with subject's pupil) at the first pupil plane after the Badal channels. The modified SimVis comprises of three main paths: The bifocal channel (Badal 1+2, SLM), the psychophysical channel (DLP) and the pupil monitoring channel. The pupil plane is conjugated with the SLM with a x0.5 magnification factor and the psychophysical is conjugated to the pupil with a $\mathrm{x} 2$ magnification factor by a pair of relay lenses of focal lengths $200 \mathrm{~mm}$ and $100 \mathrm{~mm}$. A stop placed in front of the SLM at the focus of the conjugate lens helps in eliminating the diffractive orders introduced by the SLM. An on-bench view of the modified SimVis is shown in figure $2 \mathrm{~B}$.

\section{2.c. Portable Simultaneous Vision simulator}

We developed and validated a see-through, hand-held simultaneous vision simulator using an optomechanical tunable lens. We later developed a binocular setup with similar working principles. Figure 3 shows the schematic of the miniaturized SimVis. The principal component of the system is a tunable lens (EL-10-30-C, Optotune Inc), which is a combination of polymer membrane with optical fluid that changes its curvature when a voltage is applied. Combined with an offset lens, the tunable lens has a refraction range of $-1.5 \mathrm{D}$ to $+10 \mathrm{D}$ with an effective aperture of $10 \mathrm{~mm}$. The tunable lens with an artificial pupil is placed conjugate to the exit pupil plane of the eye using a pair of achromatic doublets of $75 \mathrm{~mm}$ focal length. Image reinversion is achieved by using 4 mirrors that act as roof prism. The system has a magnification of $\mathrm{x} 1$ and a field of about 14 degrees. Multifocality is achieved by temporal multiplexing of the tunable lens to different refractive states. In our system, the transition of refraction states occur at 60 $\mathrm{KHz}$. The visual system integrates these images over time and perceives it as a static simultaneous image. The energy dedicated to a specific distance is determined by the amount of time the lens remains in that state. Thus, by modifying the time and the refraction states, bifocal and trifocal pure simultaneous images of different far/near energy ratios can be optically simulated. The tunable lens is controlled for generating refractive states by custom routines written in Visual C. Refractive errors in the eye could be compensated by the tunable lens.

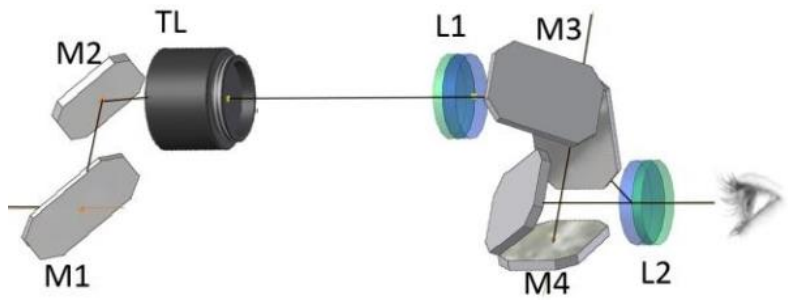

Figure 3: Schematic of SimVis mini

Figure 4A shows the miniaturized Simultaneous Vision Simulator. The binocular system (SimVis Bino) consists of two identical channels with optical design similar to the monocular system, with the exception of image inversion being achieved by a roof-pechan prism placed in front of the tunable lens expanding the field of view to 20 degrees. The tunable lens in both the channels are synchronized to perform with a desired state at the same time. The laboratory prototype of SimVis Bino is shown in figure 4B. The interpupillary distance and convergence can be changed by moving the channels lateral to the optical axis and by rotating the channels along the optical axes.

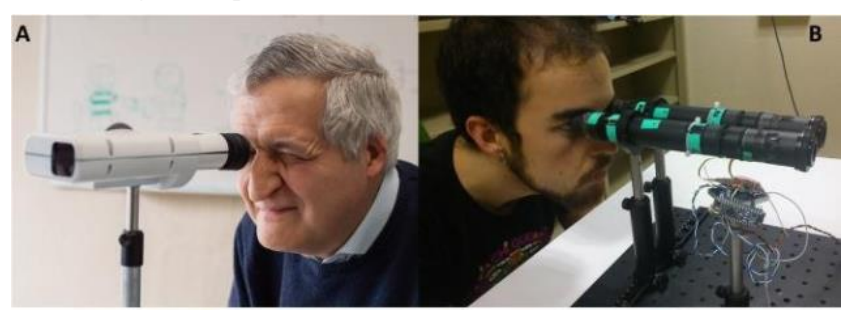

Figure 4: Simultaneous vision simulators based on temporal multiplexing (A) See-through portable monocular SimVis Mini (B) Binocular, open-field vision simulator 


\section{Measurements and Results}

\section{3.a. Adaptation to pure simultaneous vision}

A series of face images (480 $\mathrm{x} 480$ pixels) was numerically manipulated to simulate a range of pure simultaneous vision (SV) and pure defocus (PD) images. For simulating pure defocus, the face image was convolved with a point spread function of corresponding blur magnitude. For pure simultaneous vision, these images were added with a sharp image with different energy proportions. For example, a $1 \mathrm{D} 25 \mathrm{~S}-$ 75D has a sharp image (weighted 25\%) added to a $1 \mathrm{D}$ defocused image (weighted 75\%). These images were shown on a CRT monitor viewed through the adaptive optics system, to study short-term adaptation to, and perceived image quality of simultaneous vision images. Four subjects performed the measurements with their right eye. To ensure that all subjects had identically blurred images on the retina, the ocular aberrations of the subjects were corrected.

Two experiments evaluated the perception and adaptation to PD and to SV images by measuring the changes in the Perceived Best Focus and in Perceptual Score. Perceived Best Focus (PBF) is the amount of blur that produces a visual perception of neither blur nor sharp. A change in the PBF after exposure to a new visual experience (also called after-effect) accounts for a renormalization of the visual response, so that the adapting stimulus itself appears more neutral, and represents a measure of the short-term adaptation to the new extrinsic context $[27,28]$. For the PBF measurements subjects employed a single stimulus blur detection task for each of the presented test image and the blur in the next image was determined by QUEST-an adaptive psychometric procedure. The PBF was measured for 29 adapting conditions: PD images (6 different levels of defocus between 0.2 and 1.2 D), and SV 25S-75D, 50S-50D and 75S-25D images (7 near additions between 0.2 and $1.5 \mathrm{D}$ for each proportion) as well as sharp adaptation condition (defocus $=0$ ) and neutral adaptation with a gray field. The PBF shift was calculated as the difference in the PBF of the adapting image from the PBF after adaptation to a sharp image, equating a $0 \mathrm{D}$ adaptation to a 0 D PBF thereby providing a common reference for all subjects and conditions.

Results were highly consistent across subjects, with slight variations in the magnitude of PBF shifts. Figure 5 shows the average PBF shifts across all subjects. For PD adapting images, PBF shift increased up to $0.4 \mathrm{D}$, until saturation. For SV adapting images the PBF shift increased with the magnitude of defocus in the adapting images, reaching a maximum of $0.4 \mathrm{D}$ for $50 \mathrm{~S}-50 \mathrm{D}$ adaptation and $0.8 \mathrm{D}$ for $25 \mathrm{~S}-75 \mathrm{D}$, and then decreased significantly $(\mathrm{p}<0.01)$ for higher defocus values. The area under each average PBF shift curve was used to evaluate the neural adaptation for each adapting condition. There was a highly significant correlation $(\mathrm{r}=0.99, \mathrm{p}<0.001)$ between the area under the PBF shift curve and the proportion of defocus component in the adapting images (e.g.: 1 for PD and 0.50 for 50S-50D).

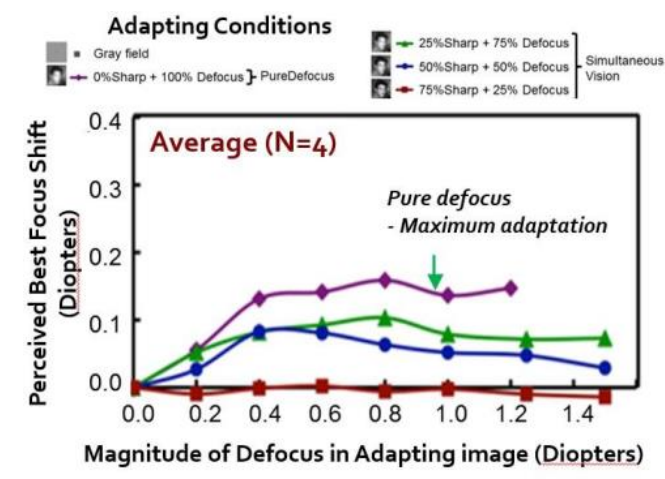

Figure 5: Average Perceived Best Focus shift after adaptation to pure Defocus and pure Simultaneous vision

Perceived image quality was measured as Perceptual Score where the subject graded the test image in a blur-sharp scale. In this experiment, subjects scored 18 PD images and 19 SV images from very sharp (score 5) to very blurred (score 0) according to the perceived quality, for 6 PD and adapting conditions: 6 different PD images (defocus ranging from 0 D to 1.2 D); and 7 different 50S-50D SV images (magnitude of defocus ranging from $0 \mathrm{D}$ to $2.5 \mathrm{D}$ ).

Figure 6 shows the average Perceptual Score of the images as a function of the magnitude of defocus in the test images for PD images (A) or in the defocused component of SV images (B). For PD images, increasing the magnitude of defocus in the test image progressively decreased the Perceptual Score. The curves shifted consistently towards higher scores following adaptation. Unlike with PD images, scoring of the SV 50S-50D test images (Figure 6B) did not decrease progressively with the magnitude of defocus (near 
addition). While there was a progressive decrease in perceived quality for simultaneous images when near addition increased from 0 to 0.4-0.5 D, the perceived quality increased for higher amounts of addition in the image. Beyond 1.5 D of addition the images were scored above 3, i.e. in the sharp region. As the blur amount increases, the blurred component of the image tends to become gray, diminishing its impact on the image degradation.

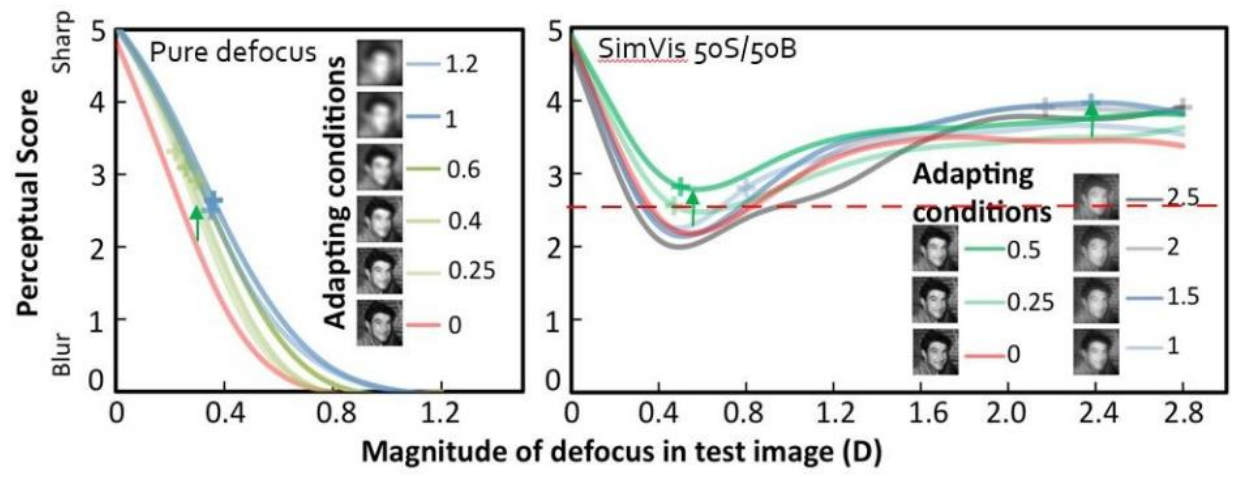

Figure 6: Average Perceptual score (A) Pure Defocus and (B) Pure Simultaneous vision

We found that, for pure simultaneous vision, the PBF shifts and the perceptual scores were correlated to image quality metrics such as RMS contrast $(r=-0.34, p=0.02)$ and MSSSIM [29]( $r=-0.54, p=0.006)$ indicating that adaptation to pure simultaneous vision is driven by retinal image contrast, similar to pure defocus adaptation.

\section{3.b. Adaptation to interocular differences in blur magnitude and orientation}

To study interocular differences in blur magnitude and orientation between eyes, PBF, after-effects and internal code for blur were measured in three separate experimental sessions. All measurements were performed monocularly in both eyes of normal subjects with undilated pupils. Sighting dominance was established in subjects using the Miles test [30]. Measurements were performed using the adaptive optics setup.

Ten young normal subjects (22 to 42 years of age) participated in the study. Five of the twelve subjects had prior experience in performing psychophysical measurements. The wavefront aberration maps, Point Spread Function and the corresponding Strehl Ratios (SR) are shown in figure 7. Subjects S1-S5 had similar orientation and different blur magnitude, S6, S7 had similar orientation and magnitude, S8, S9 had different orientation and similar magnitude and S10, S11 had different orientation and magnitude and S12 had mirror symmetric PSFs between eyes.

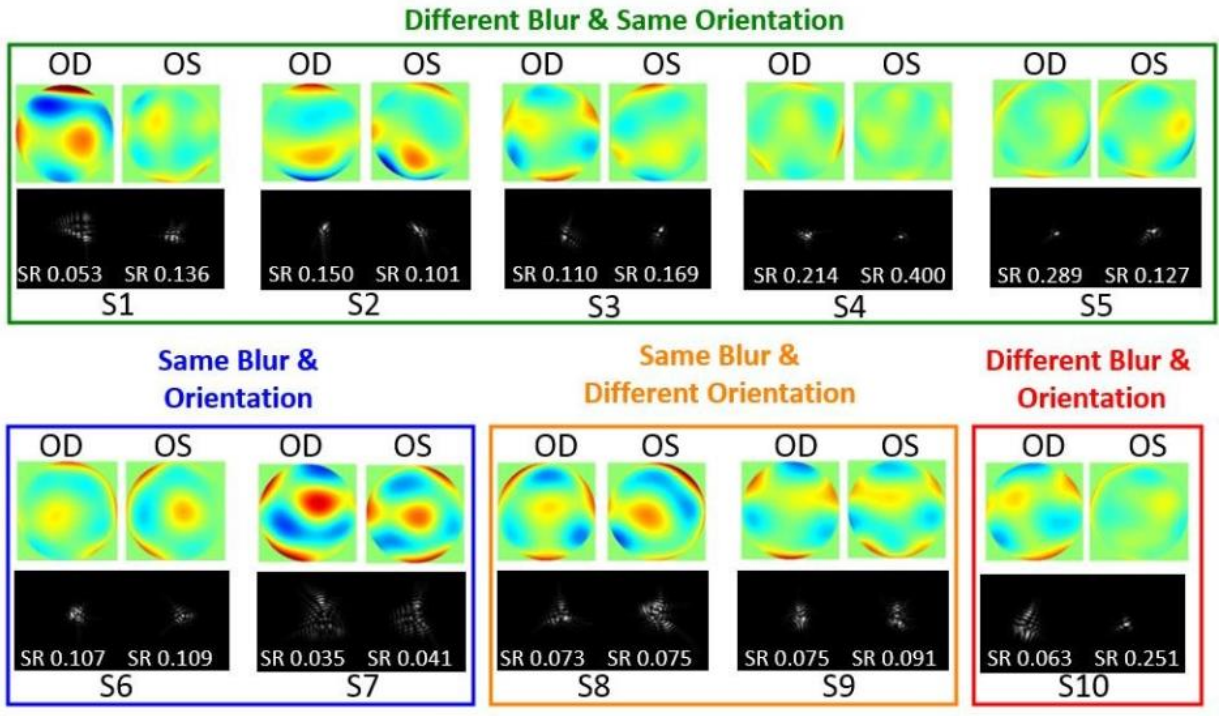

Different blur: $>30 \%$ difference in $S R$, different orientation: $>20$ deg

Figure 7: Aberration profile of subjects and ocular Point Spread functions (PSFs) 
The contour and orientation of the PSF was calculated by methods described by Sawides and colleagues [17]. The PSFs were centered at the center of mass and then sampled in 72 angular sectors of 5 degree each. The intensity of the PSF at mid-angle of each sector was obtained, was normalized to the maximum intensity and was plotted in a polar plot generating a contour diagram. The orientation axis of the PSF is given by the main axis of the best-fitting ellipse.

Perceived Best Focus was measured as described before. Test stimuli were generated by applying the optical blur measured in 128 different eyes to an image of a face of 480 pixels. Subject adapted to a gray screen for 30s and a test image with a random blur magnitude (SR) was then presented for $500 \mathrm{~ms}$. According to their subjective criterion for a correctly focused image, the subject performed a single stimulus blur detection task [31]. Depending on the response, the blur in the next image was chosen by a QUEST algorithm [32-34]. PBF was the level of blur (in SR) corresponding to the average of the last 10 stimulus values that oscillated around the boundary with a standard deviation less than $0.02 \mathrm{SR}$.

We found that despite the differences in optical quality between the eyes, the judgments of image focus were very similar when viewing the images through either eye. The amount of physical blur that observers perceived as "in focus" (neither too blurred nor too sharp) was $0.197 \pm 0.095$ SR in the better eye and $0.191 \pm 0.094$ SR in the worse eye, and the difference between eyes was not significant (SR $0.01 \pm 0.007$, $\mathrm{p}=0.547)$. There was also a strong correlation $(\mathrm{r}=0.984, \mathrm{p}<0.0001)$ between the right and left eye focus judgments (Fig. 8B).
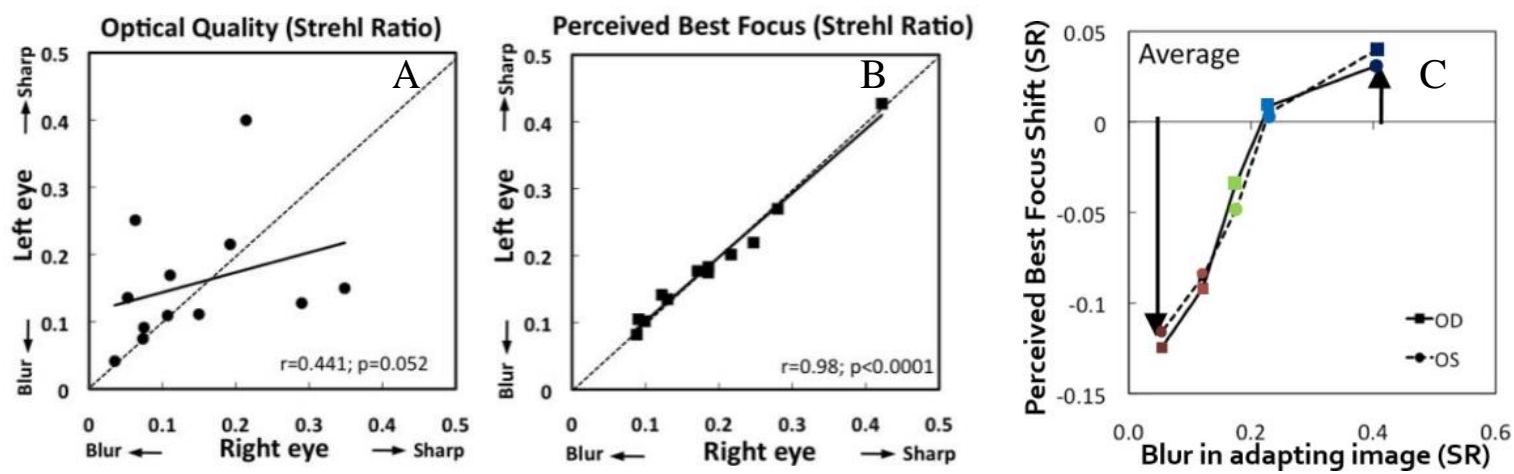

Figure 8: (A) Correlation in Optical quality between eyes ( $r=0.441, p=0.052)$; (B) Correlation of right eye PBF and left eye PBF ( $r$ $=0.984, \mathrm{p}<0.0001)$ and (C) Average shift of Perceived-Best-Focus following adaptation to different amounts of blur

The subjects (S1-S5) that had the strongest differences in blur between their eyes performed measurements on how adaptation to blur in either eye affected their focus judgments. For each subject a series of images was created that varied the magnitude of their own aberrations. To create the series, the magnitude of the PSF was scaled by a factor from 0 to 3 times the original PSF in steps of 0.01 , yielding 301 different levels. A face image was then convolved with each of these scaled PSF's to generate the image series, which ranged from no blur (SR of 1 ) to 3 times the blur of their better eye. Observers, judged the blur level that appeared neither too blurred nor too sharp, after adapting to the gray field and to images that had different degrees of blur chosen to bracket the magnitude of blur in their two eyes. Both the adapting conditions and the eye measured (left or right) were randomized.

The natural adaptation settings were strongly affected by adaptation to images with different blur levels. Figure $8 \mathrm{C}$ shows the after-effects following adaptation for all subjects and averaged across subjects. The sharpest adapting images (i.e. less blurred than the better eye) caused the blur level that was rated best focused under gray adaptation to appear too blurred. Subjects thus chose a less blurred image to compensate for this bias. Alternatively, adaptation to the most blurred image (i.e. more blurre d than the eye with poorest image quality) induced the opposite after-effect. Notably, for either eyes, the blur level at which the after-effect was nulled corresponded closely to the better eye, while exposure to the worse eye's blur or the average blur of the two eyes caused the previous subjective focus level to appear too sharp. A non-parametric ANOVA further revealed that there was no difference in the magnitude of the effects between the right and left eye $(\mathrm{p}=0.819)$. Thus both the focus judgments and how they were biased by the adaptation were completely determined by the better eye, and were consistent with a neural calibration matched to the optical quality of the less aberrated eye.

The orientation preference was performed in all subjects using classification images technique [17]. Subjects were presented 50 random pairs of images blurred from 100 different PSFs. The subject chose the better perceived image in the pair in a confidence scale of 1-3. To estimate the neural PSF, PSFs 
corresponding to the images that were subjectively selected as better-perceived are given positive scores, and the other image in the pair is given negative scores. The PSF intensities are multiplied by a score derived from the confidence score. A score of 10 is given for a very confident response, a 5 to a less confidence response, and a score of 1 is given for the lowest confidence response to each image of the pair selected as better focus. Alternatively, scores of $-10,-5$ and -1 are given to the images not selected as better focused. All the weighted responses were then summed to obtain a pattern classification map [17, 35]. The contour of the positive weights of the classification map was termed as positive neural PSF and the contour of the negative weights of the classification map was termedthe negative neural PSF.

Figure 9 shows the example of classification maps for a representative subject from each group. It could be seen that the orientation of the positive (green) and negative (red) neural PSFs was strikingly similar between the two eyes, despite similar or different optical blur magnitude and/or ocular PSF orientation (blue). There was strong and significant interocular correlation in the orientations of the positive neural PSF ( $\mathrm{r}=0.95, \mathrm{p}<0.001)$ and negative neural PSF $(\mathrm{r}=0.99 ; \mathrm{p}<0.001)$. Furthermore, the neural PSF orientations were not statistically significantly different between eyes $(\mathrm{p}=0.9$ and $\mathrm{p}=0.36$, for positive and negative, respectively). Across all subjects, the average difference in orientation between the positive and the negative neural PSFs was $58 \pm 18.73$ deg and was statistically significant ( $t$ score $=2.82, d f=9, p=0.022$ ).

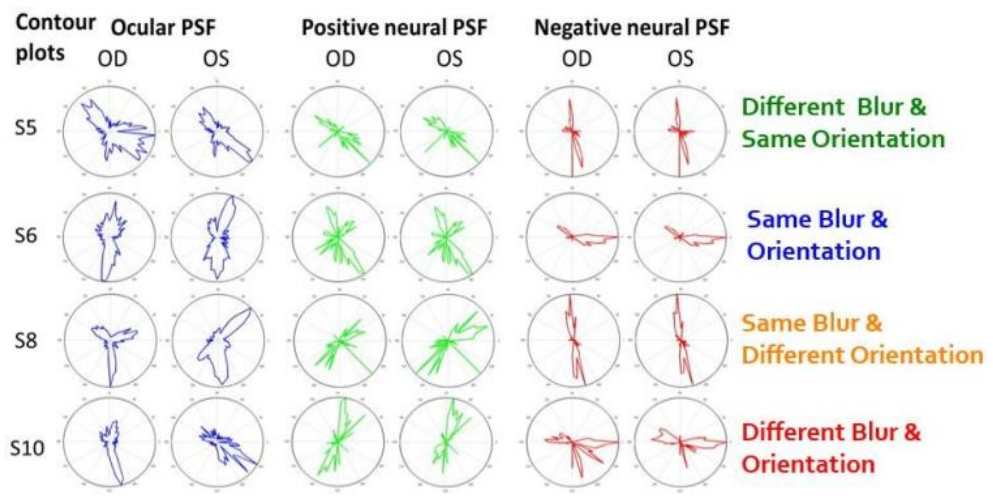

Figure 9: Ocular PSF contours (blue, left columns), positive neural PSF contour (green, middle columns), negative neural PSF contour (red, right columns) for both eyes

\section{3.c. Perception to segmented bifocal patterns}

The psychophysical measurements were performed by optically simulating simultaneous vision using a modified version of the simultaneous vision simulator $[13,26]$ incorporated with a transmitting SLM (LC2002, Holoeye Inc) conjugate to the pupil plane was used to introduce pupil phase patterns. Images were prejected at the retinal plane using a pico projector. Five normal subjects (ages $28-42$ years) participated in the measurements. A gray scale face image ( $98 \times 98$ pixels) was used as the visual stimuli and spatial sampling of different pupil patterns were facilitated by the transmitting SLM which acts as a LCD and a linear polarizer. Fourteen bifocal pupil patterns were displayed on the SLM as black and white image (as shown in the first column of figure 10). These pupil masks were generated using Matlab (Mathworks Inc). A linearly polarized light passing through these different portion of the SLM will be perpendicular polarized. A cubic beam splitter reflects or transmits the incident light, depending on its polarization angle, and therefore directs the beam passing through different areas of the pupil through the far or near visual channels of the simultaneous vision instrument. We tested six angular, six radial and two hybrid designs that had two to eight zones. Irrespective of the design and the number of zones, the energy distribution between far and near was always equal (50F/50N).

Preference score assessments were performed at far, intermediate and near distances. These distances were achieved by optically setting the Badal at different vergences corresponding to the subject's best focus. For assessing preference to a specific pattern, we used a 2AFC method with weighted choice. For the fourteen segmented bifocal patterns a combination 105 pairs were generated. The subjects were consecutively presented with the same face image viewed through a random pair of the segmented bifocal pattern. The task of the subject was to report whether the first image or the second image was better focused. A trial consisted of presentation of the 105 image pairs in random order. For far, intermediate, near distances the measurements was repeated three times, thus each segmented bifocal pattern was evaluated 45 times at any given distance. The corrections chosen by the subjects were given a positive score and the other correction in the pair was given a negative score. A Bernoulli CDF was assumed for a significance level of $5 \%$, a score point greater than 24 indicates significant preference and a score point of 
less than 14 indicates significant rejection. Thus for a given distance a score of greater than 72 indicates significant preferences and a score of less than 42 indicates significant rejection.

The preference maps for each subject at each distance and their statistical significance is summarized in Figure 10. For each subject, each column represents a testing distance and dots in green indicate statistically significant preference $(\mathrm{N}>24)$, dots in red indicate a rejection that is statistically significant and gray dots non-significant preferences for a Bernoulli CDF of $\mathrm{p}<0.05$. It is evident that radial patterns show a reversal in preference between far and near and shows a design dependence, i.e., the far score was correlated to the proportion of far area in the central $3 \mathrm{~mm}$ of the segmented bifocal pattern $(\mathrm{r}=0.82$, $\mathrm{p}<0.0001$ ). Similarly, for the same testing distance opposite preferences were observed for patterns that are symmetric about the horizontal or vertical axes (angular and hybrid designs). Despite intersubject variabilities, on an average across distances and subject the angular designs were significantly preferred and the hybrid designs were significantly rejected.

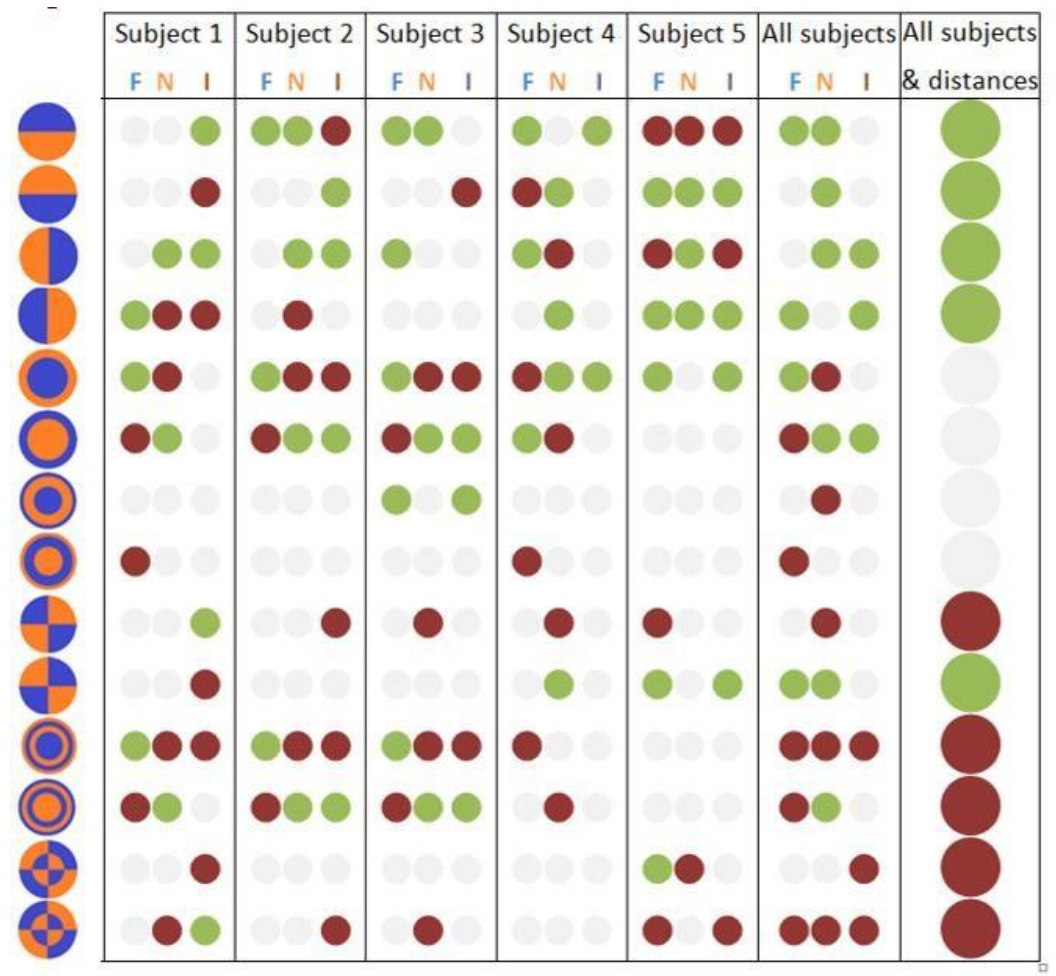

Figure 10: Preference maps for all subjects across Far, Intermediate and Near distances. Red dots indicate significant rejection, green dots indicate significant preference and gray dots indicate non-significant preferences at $\mathrm{p}<0.05$

For a diffraction limited system, the optical quality is expected/was found to be similar across designs. Optical simulation of preference to bifocal segmented patterns were performed by ranking the SR combined with the higher order aberrations of each subject. At far distance, positive responses were predicted better than the negative responses and was significant at far for $\mathrm{S} 1(\mathrm{r}=0.55, \mathrm{p}<0.05), \mathrm{S} 2(\mathrm{r}=0.54$, $\mathrm{p}<0.05)$ and $\mathrm{S} 3(\mathrm{r}=0.65, \mathrm{p}<0.05)$. At near distance the responses correlation between simulation and measurements were significant only for $\mathrm{S} 1(\mathrm{r}=0.7, \mathrm{p}<0.01)$.

\section{3.d. Preference to oriented bifocal patterns}

Subjective preference to oriented bifocal patterns were tested using the modified simultaneous vision simulator. Twenty subjects (aged 21-62 years) participated in our measurements. Using the SLM in the SimVis setup we optically simulated the design of a commercial IOL, M Plus (Oculentis Inc). The lens has an angular profile with a small radial zone at the center for far vision. The far/near energy ratio was 60/40. Using Matlab, masks for SLM were created with white portions of the image representing far vision and black portions representing near vision. The mask created with Matlab and the corresponding representation through a near channel is shown in Figure 11a. Psychophysical measurements were performed with eight orientations of the mask with angular designation corresponding to the location of near zone (Fig. 11b). 


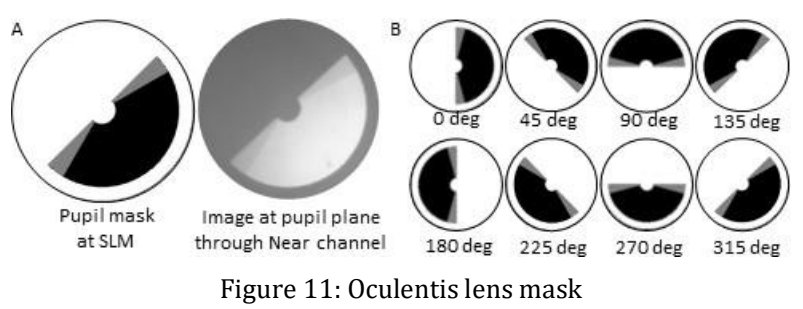

For testing the orientation preference, we employed a classification image method [35, 36]. Subjects viewed subsequently, a face image through two pairs of random orientation of the bifocal design for $1.5 \mathrm{~s}$ each with a gray screen presented in between each image pair presentation. The subject's task was to choose the better focused image (first or second) of the pair and indicate the confidence of choice on a 3 level scale. Each session consisted of presentation of 36 random pairs and the measurements were repeated 10 times. Preference scores were assigned as described in section 3.b. In addition, preferred orientation at each distance was calculated as the orientation of centroid of the polygon encompassing the data for eight orientations.

The pattern preference was also simulated using an ideal observer model. We computed the throughfocus VSOTF for each orientation of the bifocal combining it with the subject's aberrations (astigmatism+HOA). At any orientation, VSOTF corresponding to the best focus, at $+1.5 \mathrm{D}$ and at $+3 \mathrm{D}$ was compared with the other orientations and scores of 10,5 and 1 were assigned when the difference between the orientations were above $75 \%, 50 \%$ and $25 \%$ thresholds respectively.

Figure 12 shows the weighted preference for representative subjects with their respective centroids. The center of the plot corresponds to a preference score of -100 and extends symmetrically across all orientations to a score of +100 . The arrows indicate the orientation of the centroid (preferred orientation) and the length of the vector indicates the strength of preference. The trends noted in the unweighted preference were preserved in the weighted preferences. The weighted perceptual preferences correlated significantly with the perceptual score across all distances $(r=0.48, p=0.004)$. At far, 6 subjects preferred nasal quadrant $(0 \pm 44.5 \mathrm{deg}), 4$ subjects preferred superior quadrant $(90 \pm 44.5 \mathrm{deg}), 8$ subjects preferred temporal quadrant $(180 \pm 44.5 \mathrm{deg})$ and only 2 subjects preferred inferior quadrant $(270 \pm 44.5$ deg). Also at near, horizontal orientations were preferred more than vertical orientations (8 nasal and 5 temporal), with 6 subjects preferring the inferior and only one subject preferring the superior quadrant.

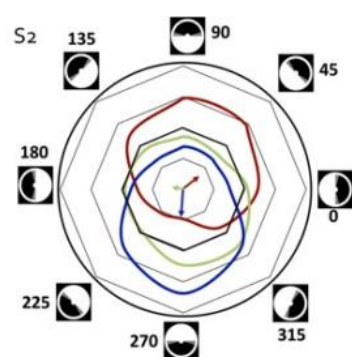

Strong preference, changes from far to ne $(N=8)$

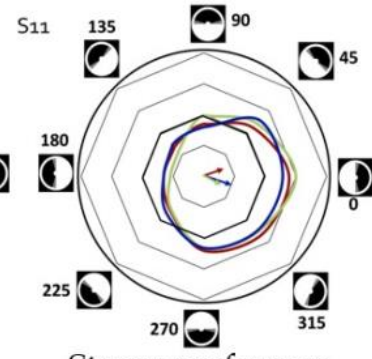

Strong preference, constant across distance $(\mathrm{N}=9)$

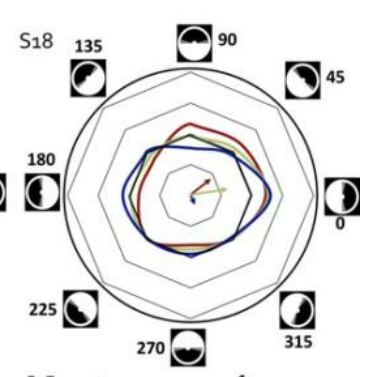

No strong preference at any distance

$(\mathrm{N}=3)$

Figure 12: Weighted orientation preference for representative subjects at far (red), intermediate (green) and near (blue) distances. At any orientation, the axes extends from +100 to -100 in the center with black line representing the zero. Arrows indicate the preferred orientation at the respective distances with the length of the arrow indicating significant preferences.

The centroid locations for far and near is shown in Figure 13. The filled symbols represent far centroids and open symbols represent near centroids. Also, subjects with right eye measured are shown in diamonds. Values outside the inner circle (radius of 15) are significant. Only 8 subjects had strong orientation preference at far and at neat only 9 subjects had strong orientation preference. The mean difference in the centroid location between far and near was $27+22$ degrees indicating that in most subjects the orientation preference was retained across distances. However, the centroid locations between far and near correlated only weakly $(\mathrm{r}=0.32, \mathrm{p}<0.05)$. 


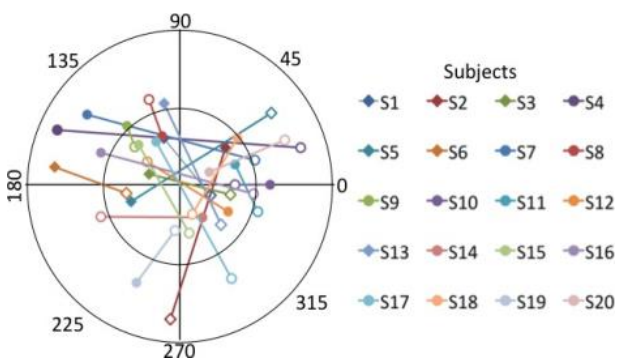

Figure 13: Far (filled symbols) and near (open symbols) centroid locations. Inner circle represents significant radius of centroids.

Figure 14 (A-C) shows the centroid locations for the measured (filled symbols) and simulated (open symbols) values at far, intermediate and near distances. It could be observed that the data are much scattered for intermediate distance. The mean difference between measurement and simulation in the centroid location was $28 \mathrm{deg}$ (SD: 29) at far, $80 \mathrm{deg}$ (SD: 63) at intermediate and $36 \mathrm{deg}$ (SD: 28) at near distances. Correspondingly, there was a strong significant correlation between the measured and simulated centroid orientations at far and near distances $\left(r_{f}=0.89, r_{n}=0.94, p<0.0001\right)$. Even at intermediate distance a weak correlation was observed $\left(\mathrm{r}_{\mathrm{i}}=0.47, \mathrm{p}<0.05\right)$. The radius of the centroid estimated by simulations were higher than measurements in about $65 \%$ of the subjects across distances. On an average across distances and subjects, the difference in radius between simulations and measurements were 1.8 (SD: 7.8) and did not correlate significantly at any distance.
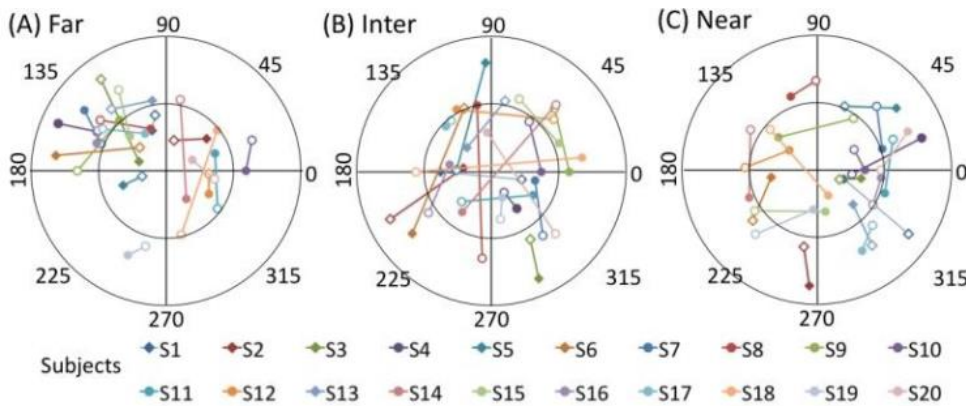

Figure 13: Centroid locations for measured (filled symbols) and simulated (open symbols) data at (A) far, (B) Intermediate and (C) Near distances for all subjects. Inner circle represents significant radius of centroids.

\section{3.d. Perceptual performance with monocular portable Simultaneous Vision Simulator}

Using the portable, see-through simultaneous vision simulator (using temporal multiplexing) three monofocal, two bifocal and two trifocal corrections were simulated using the simultaneous vision simulator. The monofocal corrections were 100\%Far (100F), 100\%Intermediate (100I) and 100\%Near $(100 \mathrm{~N})$; the bifocal corrections were $50 \% \mathrm{Far} / 50 \%$ Near $(50 \mathrm{~F} / 50 \mathrm{~N})$ and $70 \% \mathrm{Far} / 30 \%$ Near $(70 \mathrm{~F} / 30 \mathrm{~N})$; and the trifocal corrections assessed were 33\%Far/33\%Intermediate/33\%Near (33F/33I/33N) and $50 \% \mathrm{Far} / 20 \%$ Intermediate $/ 30 \% \mathrm{Near}(50 \mathrm{~F} / 20 \mathrm{I} / 30 \mathrm{~N})$. For assessing the perceptual quality, a real visual scene was simulated in a laboratory environment, with targets at far $(4 \mathrm{~m})$, intermediate $(66 \mathrm{~cm})$ and near $(33 \mathrm{~cm})$ distances for perceptual measurements. The visual scene consisted of a poster of a landscape and a high contrast letter (logMAR 1) at far, covering the upper right quarter of the visual field, a laptop with high contrast text at intermediate distance covering the upper left quadrant and a mobile phone with the same high contrast text covering the inferior zone at near distances. For near and intermediate distance the same continuous text of non-serif letters was used and the size of the letters at near was $14 \mathrm{pt}$ and at intermediate distance it was $18 \mathrm{pt}$. In total, $30 \%$ of the visual scene was dedicated for far vision, $30 \%$ for intermediate vision and 40\% near vision (Fig. 14).

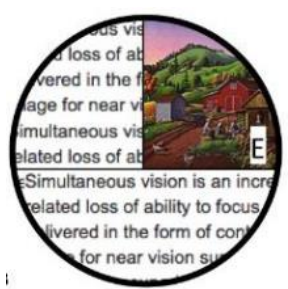

Figure 14: Perceptual preference measurements using visual scene with landscape for far and a high contrast text for intermediate and near distances. 
Nine subjects (ages 20 to 62 years) participated in the measurements. The task of the subject was to score the perceptual quality of the visual scene in a scale of 0 (very blurred) to 5 (very sharp) for each of the correction optically induced through the portable SimVis. The measurements were repeated five times and the average of the five reading were considered for analysis. The usefulness of the portable SimVis in assessing visual acuity and preference scores were also assessed, which are described elsewhere [25].

The average perceptual score varied systematically across designs (Fig. 15A). The perceived quality at far or near correlated significantly and strongly with the percentage of energy devoted to far or near in each correction $(r=0.92, p<0.0001)$. The overall perceptual score correlated significantly with the intermediate $(r=0.65, p<0.0001)$ and far $(r=0.51, p<0.001)$ perceptual scores, but not with the near perceptual score $(\mathrm{r}=-0.07, \mathrm{p}=0.57)$. On average, the overall perceptual score was maximum for the 100I correction (score $3.5 \pm 0.6$ ) among the monofocal corrections, and was maximum for $50 \mathrm{~F} / 20 \mathrm{I} / 30 \mathrm{~N}$ (score $2.7 \pm 0.6$ ) among the multifocal corrections. Figure 15B shows the perceptual scores for individual subjects for the monofocal corrections (red, green and blue bubbles represent far, intermediate and near corrections). For $100 \mathrm{~F}$, the perceptual score was 5 in all subjects at far, but for the same correction at near it ranged from 0 to 3. Similarly, perceptual score for $100 \mathrm{~N}$ ranged from 4 to 5 at near and at far it ranged from 0 to 3 . Monofocal intermediate correction showed the largest range of perceptual scores across subjects at far (1.7 to 3.3) and near (1.3 to 5) distances, indicating large intersubject variability in the responses. On the other hand, the multifocal corrections (Fig. 15C) had similar range of perceptual score at far and near distances. Specifically, the 50F/20I/30N showed the narrowest range at far ( 2 to 4 ) and 33F/33I/33N had the smallest range at near (2.1 to 4.3).

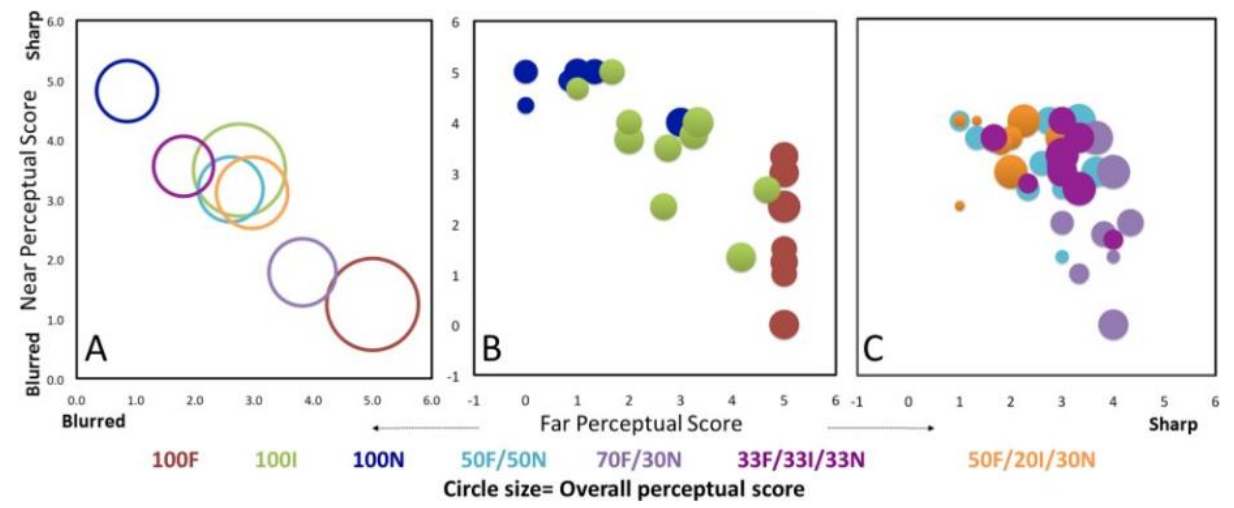

Figure 15: Perceptual score at far and near distances. Bubble size indicates overall score. (A) Average across subjects for all corrections. (B) For monofocal corrections in all subjects. (C) For simultaneous vision corrections in all subjects.

\section{3.e. Adaptation to pure simultaneous vision}

Psychophysical measurements were performed under optical simulation of binocular presbyopic corrections by synchronized temporal multiplexing of the tunable lenses in both channels of the binocular mini simultaneous vision simulator. Binocularly symmetric corrections included monofocal correction in both channels at far, intermediate and near distances $(\mathrm{F}+\mathrm{F}, \mathrm{I}+\mathrm{I}, \mathrm{N}+\mathrm{N}$ respectively). For monofocal corrections, tunable lens was placed at a static focus of $0 \mathrm{D}$ (for far), $+1.5 \mathrm{D}$ (for intermediate) and $+3 \mathrm{D}$ (for near). Binocular simultaneous vision correction were induced by multiplexing both the tunable lens at different focus states. It included, bifocal correction with 50\%Far/50\%Near (2SV+2SV), trifocal correction with $50 \% \mathrm{Far} / 20 \%$ Intermediate/30\%Near (3SV+3SV) energy distributions in both channels, and combinations of bifocal and trifocal $(2 \mathrm{SV}+3 \mathrm{SV}, 3 \mathrm{SV}+2 \mathrm{SV})$ in either of the channels.

Monovision was simulated by focusing one channel at far and the other at near $(\mathrm{F}+\mathrm{N})$. Modified monovision corrections were induced by setting a monofocal focus at far or near in one channel and setting a simultaneous vision correction in the other channel $(\mathrm{F}+2 \mathrm{SV}, \mathrm{F}+3 \mathrm{SV}, 2 \mathrm{SV}+\mathrm{N}$ and $3 \mathrm{SV}+\mathrm{N})$. For psychophysical measurements a visual scene was created with a poster and a high contrast target at far $(4 \mathrm{~m})$, a laptop at intermediate $(66 \mathrm{~cm})$ and a smartphone at near $(33 \mathrm{~cm})$ distances as in figure 14 .

Binocular fusion, through two channels, was tested and adjusted for each subject, for the three distances by placing a reticule in each channel. For each subject, the refractive corrections at far, intermediate and near distances were assessed for each eye. Eight subjects (age range 23 to 45 years) performed the pattern preference measurements. Ocular dominance was assessed using Miles test (Roth et al., 2002). The subjects viewed the same visual scene in succession through two presbyopic corrections and reported which of the two corrections provided an overall better perceptual quality. For pattern preference measurements, the dominant eye was always corrected for far and 36 pairs of the following 9 
combinations were assessed. The corrections chosen by the subjects, were given a positive score and the other correction in the pair was given a negative score.

Figure 16 shows the pooled (across subjects) pair-wise comparison preference map. Among the designs tested, the monovision corrections were preferred $97 \%$ of the times and a far dominant modified monovision $(\mathrm{F}+3 \mathrm{SV}$ or $\mathrm{F}+2 \mathrm{SV})$ were preferred about $63 \%$ of the times presented. Assuming a Bernoulli's distribution of $\mathrm{p}<0.05$, green dots indicate that the design on the vertical axis was preferred significantly over the one in horizontal axis and a red dot indicates that the design on the vertical axis was significantly rejected compared to the one in horizontal axis, while a gray dot indicates non-significant preferences. As reflected in the percentage preferences, monovision and modified monovision (far or near dominant) corrections were preferred in general by all subjects. Interestingly, on an average across subjects, the binocular bifocal $(2 \mathrm{SV}+2 \mathrm{SV})$ pattern was significantly preferred over any other binocular simultaneous vision corrections and the other simultaneous vision corrections were neither preferred nor rejected significantly (except binocular trifocal corrections).

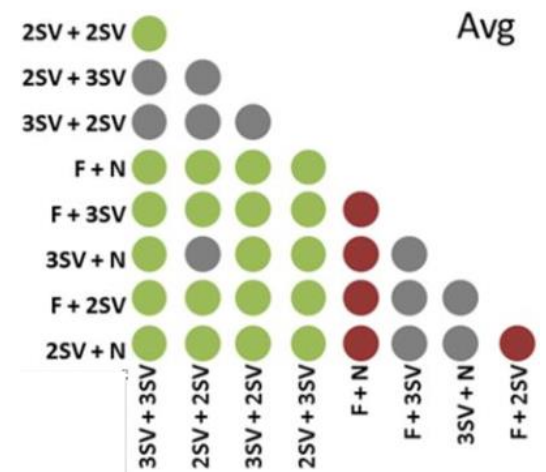

Figure 16: Preference maps for pair-wise comparison of binocular simultaneous vision, monovision and modified monovision corrections on an average across subjects

\section{Conclusions}

The Perceived Best Focus shifts after adaptation to both pure defocus and pure simultaneous vision. The change in Perceived Best Focus correlated strongly to the magnitude (amount of near addition) and the proportion (far/near ratio) of blur in the image. Maximum perception of blur and maximum adaptation to simultaneous vision was noted for low near additions ( 0.50 D).Mechanism of perception of/adaptation to simultaneous vision is found to be similar to that of monofocal blur driven by contrast adaptation. In subjects with different blur magnitude between eyes, what people perceive as 'best-focused' matches the blur encountered in the eye with better optics, even when judging the world through the eye with poorer optics. In subjects with different blur magnitude and orientation, the orientation of the blur perceived as better focused correlated with the orientation of blur in the eye with better optical quality. The orientation of positive neural PSF was about 60 degrees apart from the orientation of negative neural PSF. The internal code for blur is the same for both eyes, in both magnitude and orientation, even under binocular dissociation, suggesting a cyclopean locus for blur adaptation in the higher cortical regions. Subjects preferred strongly the blur introduced by an angular, two segmented bifocal design. Radial designs were neither significantly preferred nor rejected. Hybrid designs were strongly rejected by the subjects. Preferences changed across distances and across subjects. For an angular bifocal design, preferences changed with change in orientation of the bifocal design and across distances. Most subjects preferred horizontal orientation of the near segment. Subjective orientation preference to the bifocal angular design was predicted by optical metrics, combining the bifocal design and ocular aberrations. The hand-held SimVis (monocular and binocular) based on temporal multiplexing is an effective tool to optically simulate multifocal correction enabling multifocal prescription evidence-based and by providing subjects with firsthand information on multifocality. We found that visual and perceptual performances are affected to a great extent by the far/near energy ratio and by visual needs of the subject. Simultaneous vision corrections provided uniform and acceptable visual performances at all distances. Binocularly, subjects preferred monovision and modified monovision corrections (monofocal + simultaneous vision combinations) compared to binocular simultaneous vision corrections. 
ÓPTICA PURA Y APLICADA

www.sedoptica.es

\section{Acknowledgements}

The authors have commercial interest in the simultaneous vision simulator used in the study. The authors acknowledge the funding received from the European Research Council under the European Union's Seventh Framework Programme (FP7/2007-2013) / ERC Grant Agreement no 294099 (SM), Spanish Government grant FIS2011-264605 and FIS2014-56643-R (SM), the 7th Framework Programme of the European Community through the Marie Curie Initial Training Network OpAL (OpAL is an Initial Training Network funded by the European Commission under the Seventh Framework Programme (PITN-GA2010-264605, AR). 